\title{
Plasma Catecholamine's and Blood Pressure Changes after Prolonged Exposure to Stressful Stimuli (Heat)
}

\author{
Dr. Jagdish C Hundekari ${ }^{1}$ \\ ${ }^{I}$ Associate professor, Department of Physiology, MIMER Medical College, Pune Maharashtra University of \\ Health Science, Nasik, Maharashtra (India)
}

\begin{abstract}
Abstarct: The aim of this study was to evaluate the relationships between heat stress and indicators of autonomic nervous system activity in workers of the Thermal Power Station. A total of 123 employees between 12-58yrs of age from a company that produces electricity were recruited for participation in this study. Thermal stress was assessed in workers exposed to heat to temperature between $38^{0} \mathrm{C}$ to $43^{\circ} \mathrm{C}$ in Boiler section. The workers were divided into four groups depending on duration of exposure. Systolic and Diastolic Blood pressure was recorded and plasma catecholamine's concentration was measured by an HPLC-ECD. Information on demographic characteristics, previous job history, smoking status and alcohol consumption was also collected. Blood pressure was significantly higher with prolonged duration of stressful (heat) stimuli.The plasma catecholamine's levels increases significantly. The results of the present study indicate that these modifications are related with the duration of exposure to heat. It is presumed that these effects are due to activation of autonomic nervous system and Hypothalamo-Hypypophyseal adrenal axis and resultant release of catecholamine's from adrenal medulla due to heat stress. These suggest that the risk of hypertension and Coronary Heart Disease (CHD) is greater in these workers.
\end{abstract}

Key words: heat stress, systolic Blood Pressure, Diastolic Blood pressure plasma epinephrine, plasma norepinephrine, CHD

\section{INTRODUCTION}

Stress may be defined as "non specific result of any demand upon the body". It can be mental or physical. ${ }^{[1]}$ Stress can be considered as state of disharmony or threatened Homeostasis. Stress conditions vary and can range from physical to psychological, from mild to severe and from acute to chronic. ${ }^{[2]}$ Cardiovascular responses during and after psychological stressful situation have been frequently investigated with an objective to correlate behavioural and physiological aspects of stress ${ }^{[3]}$. Several kind of mental stress are associated with temporary rise in blood pressure and plasma catecholamine's ${ }^{[4,5,6,7]}$ Previous studies have revealed significant relation between occupational exposure to noise and cardiovascular changes ${ }^{[\mathbf{8 , 9}]}$ but there is little information about the association between occupational exposure to heat and cardiovascular changes. Fire is one of the most serious problems that affect the modern life. The workers are entrusted with important task of firing the boiler in boiler section. So, they are continuously exposed to heat emitted by the boilers. This job is stressful and thus prompted us to study the influence of varying duration of heat exposure on plasma catecholamine's and blood pressure. The employees work round the clock in shifts and studies have shown that workers employed in three shift jobs suffer more from work-related stress than do day workers. ${ }^{[10]}$

\section{MATERIAL AND METHOD}

\subsection{Subjects}

The subjects were 123 male healthy employees in between age group of 21-58 yrs from a Thermal power plant that produces electricity. Only males were included in the study as the company employs very less females. The employees work one of three shifts (morning, afternoon or night). The workers were exposed to heat emitted by boiler in boiler section for 8hours daily and six days a week. Temperature to which they are exposed is in between $38^{0} \mathrm{C}$ to $43^{\circ} \mathrm{C}$. Self-administered questionnaires were used to gather information on demographic characteristics, job history, smoking history and alcohol consumption. All the subjects selected were free from diabetes, cardiovascular or other systemic disorders. Also the smokers and alcoholic were excluded.

The workers were grouped into four Groups depending on duration of exposure as shown in Table No.1

\begin{tabular}{|c|c|c|c|c|}
\hline Groups & I & II & III & IV \\
\hline Age (yrs) & $21-30$ & $31-40$ & $41-50$ & $51-58$ \\
\hline $\begin{array}{c}\text { Duration of } \\
\text { Exposure (yrs) }\end{array}$ & $5-10$ & $11-15$ & $16-20$ & $>20$ \\
\hline No. of Workers & 16 & 42 & 45 & 20 \\
\hline
\end{tabular}




\subsection{Plasma Catecholamine Assay}

To obtain quantitative measurements of autonomic nervous system response, epinephrine and nor epinephrine levels were measured from a single $5 \mathrm{ml}$ venous blood sample, collected into plasma blood tubes after obtaining a informed consent. Participants were instructed to fast 2 hours prior to test and blood sample were collected in supine position (30mins) at same time of the day to minimize circadian and diurnal fluctuations in the biomarkers. Plasma was mixed and spun, serum was allowed to clot for 30 minutes and they were then centrifuged for 10 minutes. The quantitative measurement of epinephrine and norepinephrine were assayed by means of HPLC with fluorimetric detection after isolation from plasma by a specific liquid/liquid extraction method. ${ }^{[11]}$

\subsection{Blood pressure measurement}

Because large variations in blood pressure have been shown to exist with a given subject, it is difficult to detect differences between groups that may lead to misclassification of individuals. Therefore, multiple blood pressure measurements using standard procedures were implemented in this investigation. Their blood pressure was recorded in supine position using mercury sphygmomanometer. Three readings were taken 10minutes apart and mean was taken as blood pressure.

\subsection{Statistical analysis}

Statistical comparisons between various groups were made by analysis of variance (ANOVA). ANOVA was performed in order to evaluate the difference in blood pressure and plasma catecholamine's in the four groups according to the duration of exposure to heat.

\subsection{Prevalence of hypertension}

\section{RESULTS}

The cardiovascular parameters measured in workers exposed to heat depending upon duration of exposure is demonstrated in Fig. No.1 \&2. When the workers were categorized into four groups depending upon duration of exposure, systolic and diastolic blood pressure increases significantly as duration of exposure to heat increases.

Fig. No.1 :-Changes in systolic Blood Pressure amongst the workers depending upon duration of exposure.

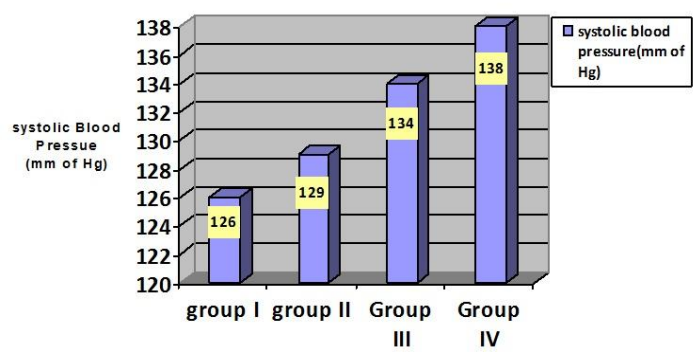

Highly significant increase in systolic blood pressure is observed in fig. no.1 as duration of exposure to heat goes on increasing form group I to group IV in workers.

Fig. No.2 :-Changes in diastolic Blood Pressure amongst the workers depending upon duration of exposure.

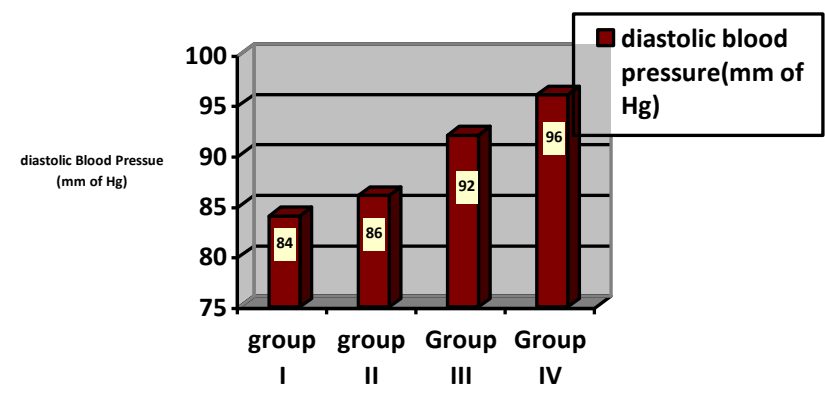


Significant increase in diastolic blood pressure is observed in fig. no.2 as duration of exposure to heat goes on increasing form group I to group IV in workers.

3.2 Changes in the Plasma Catecholamine's

Figure No.2 summarizes the results of occupational studies on the association between heat and stress hormones. When the levels of plasma catecholamine's such as epinephrine and nor epinephrine were compared in each group depending on duration of exposure to heat, there is significant rise in plasma catecholamine's as the duration of exposure goes on increasing.

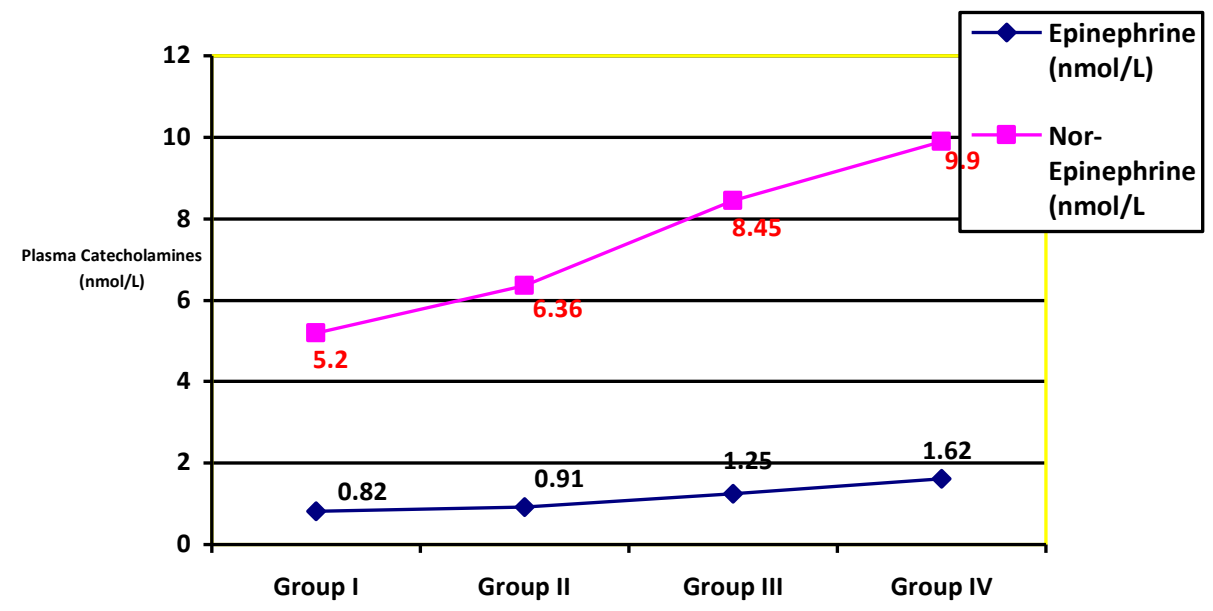

Figure No. 2 :-plasma Catecholamine's levels amongst workers with respect to duration of exposure to heat.

\section{DISCUSSION}

The present study indicates an influence of prolonged exposure to heat on the blood pressure. The primary finding of this study is that autonomic responses during occupational exposure to heat are significantly altered by heat stress.

In the present study, systolic and diastolic blood pressure showed significant increase after prolonged exposure to heat stress. In previous studies, blood pressure increase in acute heat stress in both animals and humans has been described. ${ }^{[12,13,14]}$ Parvizpoor et al ${ }^{[15]}$ conducted a study of noise exposure and prevalence of high blood pressure among 821 Iranian weavers in a textile mill. A clear increase in the prevalence of both borderline and definite hypertension was observed in relation to length of employment, an increase over and above would be due to age alone. Kavoussi et al ${ }^{[16]}$ in a study of 465 males occupationally exposed to noise observed that hypertension increases with increase years on the job. Hypertension was more prevalent among men aged 55-64 with 25 or more experience. Anders Jonsson et al(1977) ${ }^{[17]}$ and Talbott et al (1985) ${ }^{[18]}$ in their work on prolonged exposure to stressful stimuli (noise) suggested that continues exposure to stressful stimuli may have caused repeated cardiovascular reactions to psychological rise in blood pressure. A similar hypothesis putforth by Andrew Steptoe et al $(1986)^{[19]}$ that autonomically mediated cardiovascular reactions to psychosocial challenges may initiate progression towards sustained blood pressure in susceptible individuals. Saha $S$ et al (1996) suggested that the rise in blood pressure with increased duration of exposure to noise is attributed to increase release of plasma catecholamines. ${ }^{[20]}$

In our study, the rise plasma catecholamines concentration become more significant with increasing duration of exposure to heat. A similar statistical significant increase in plasma and urine levels of catecholamines was reported by Kyoung-Ho LEE et al (2010) ${ }^{[21]}$ Dimsdale JE et al(1980) ${ }^{[22]}$ Fibiger W et al $(1988)^{[23]}$ Chang HR et al (1996) ${ }^{[24]}$ in acute and chronic cases of different types of stresses.

Norepinephrine secreted by sympathetic nerve endings binds to receptors on neighbouring organs and influences their function and then undergoes re-uptake at the sympathetic nerve endings. Epinephrine and norepinephrine secreted by the adrenal medulla enter the bloodstream and exert their effects on organs farther away. These hormones work by stimulating the $\alpha$ and $\beta$ receptors, each having a specific effect at each receptor type. Adrenal medulla also secretes epinephrine and norepinephrine as well. Epinephrine secretion can, therefore, be used as a reflex of sympathetic nerve function, as well as adrenal medulla function. In addition, catecholamine increases can be seen in essential hypertension, malignant hypertension, and congestive heart failure, but catecholamine monitoring in these diseases is not diagnostically useful. Under stress, catecholamine levels can also be increased. ${ }^{[25]}$ 


\section{Conclusion}

From the present study it may thus be concluded that heat stress causes increased sympathetic activity thereby leading to significant increase in systolic and diastolic blood pressure. It can also be concluded that prevalence of hypertension, CHD and its risk is higher in workers exposed to heat as a stressful stimuli for longer duration

\section{Acknowledgements}

This project was funded and supported by MIMER Medical College. The authors wish to acknowledge the Director and the staff of Thermal Power Station (Parli, Maharashtra, India) for support and assistance provided during study. We are extremely grateful to Dean, MIMER Medical College for permitting me to undertake this study in the Department of Physiology.

\section{References}

[1] Singhal S. et al. Effect of immobilisation stress on lipid profile. Indian J. of phy. and allied science 1997;51(3)):138-143.

[2] H.S.Nagaraja et al. Influence of different types of stress on selected cardiovascular parameters in rats IJPP 1999;43(3) : 296-304).

[3] Koolhaas JM et al Coping statergies and Cardiovascular risk : A study of rats and mice in Behavioural observations in cardiovascular research. Amsterdam; $1999: 45-60$.

[4] Tulen JH et al -CVS control and plasma catecholamines during rest and mental stress : effect of posture. Clinical science $1999 ; 96(6): 567-76$,

[5] Crandal CG et al Cardiovascular function in the heat-stressed human. Acta Physiol 2010 Aug;199(4):407-23.

[6] Henry JP et al Psychological stress can induce chronic hypertension in normotensive strains of rats. Hypertension.1993;21;714723.

[7] Herd JA et al - cardiovascular response to stress. Physiol Rev 1991;71:305-330

[8] Turkhan JS et al Novel long-term cardiovascular effects of noise stress. Physiol Behave. 1984 Jul;33(1):21-6.

[9] Peterson EA et al. Daily noise duration influences cardiovascular responses. J Aud Res 1984 Apr;24(2):69-86 )

[10] Harada H et al .Three-shift system increases job related stress in Japanese workers. J. occupational Health 2005;47: 397-404.

[11] Davies CL et al. Routine determination of plasma catecholamines using HPLC with electrochemical detection.J Chromatography $1982 ; 231: 41-51$.

[12] Scott Let al. Heat stress alters hemodynamic responses during the Valsalva manoeuvre. J applied physiology $2010 ; 108$ : 1591 1594

[13] M. J. Gollock et al Cardiovascular and haematological responses of Atlantic cod (Gadus morhua) to acute temperature increase. The Journal of Experimental Biology 209, 2961-2970.

[14] Jian Cui et al Heat stress attenuates the increase in arterial blood pressure during the cold pressor test. J Appl Physiol 2010 ; 109 : $1354-1359$.

[15] Parvizpoor D et al (1984). Noise exposure and prevalence of high blood pressure amongs weaver in iran. J. occupational medicine ; $18: 730-1$.

[16] Kavoussi et al The relationship between the length of exposure to noise and the incidence of hypertension at a silo in Tehran. Med Law 1973;64:292-295.

[17] Anders Jonsson et al. Prolonged exposure to a stress stimuli(noise) as a cause of raised blood pressure in man. Lancet 1977; January 8: 186(7)

[18] Talbott et al. Occupational noise exposure, noise induced hearing loss and the epidemiology of high blood pressure. American J. of epidemiology $1985 ; 120(8): 501-514$.

[19] Andrew Steptoe. Stress mechanism in hypertension. Postgraduate medical journal1986 ; 62: 697-699.

[20] Saha S et al. Effect of noise stress on some cardiocascular parameters and audiovisual reaction time. IJPP1996;40(1):35-40.

[21] Kyoung-Ho LEE et al .Heart Rate Variability and Urinary Catecholamines from Job Stress in Korean Male manufacturing Workers According to Work Seniority. Industrial Health 2010; 48: 331-338.

[22] Dimsdale J E et al. Plasma catecholamine in stress and exercise. JAMA 1980; $243: 340-342$.

[23] Fibiger W et al. Relationship between catecholamines in urine and physical and mental stress. Int. journal Psychophysiology 1984; 1 : 325-333.

[24] Chang HR et al. Lymphocyte beta2-adrenergic receptors and plasma catecholamine levels in lead-exposed workers Toxicol Appl Pharmacol. 1996 Jul;139(1):1-5.

[25] Young-Hee Lee et al. The Effects of Heat and Massage Application on Autonomic Nervous System , 2011Yonsei Med J ; 52(6):982-989. 